increased with the number of AEDs used: $1.5 \%$ in untreated controls, $3.3 \%$ with 1 AED, $4.7 \%$ with $2,4.4 \%$ with 3 , and $8 \%$ with exposure to $4+$ AEDs. Risk of major congenital abnormalities in the offspring was significantly increased for 1) carbamazepine and valproate monotherapy, with a significant dose-response relationship for valproate; 2) phenobarbital, when combined with caffeine; and 3) several polytherapy regimens, including clonazepam with other AEDs, carbamazepine and valproate combination, and phenobarbital with caffeine combined with other AEDs. Valproate monotherapy is especially associated with spina bifida and hypospadias. Phenytoin monotherapy was not associated with increased risk. (Samren EB, van Duijn CM, Christiaens GCML, Hofman A, Lindhout D. Antiepileptic drug regimens and major congenital abnormalities in the offspring. Ann Neurol Nov 1999;46:739-746). (Respond: Prof Lindhout, MGC-Department of Clinical Genetics, Erasmus University Rotterdam, PO Box 1738, NL-3000 DR Rotterdam, The Netherlands).

COMMENT. Polytherapy with AEDs is more often associated with an increased risk of major congenital abnormalities than monotherapy, and the risks are higher. Combinations of benzodiazepines with valproate and carbamazepine are especially teratogenic, and should be avoided during pregnancy. Caffeine, usually considered relatively harmless, raises the risk of malformations if taken in combination with phenobarbital during the first trimester. The ingestion of caffeine containing drinks might also pose a risk factor.

\title{
CEREBELLAR ATAXIA
}

\section{CEREBELLAR ATAXIA WITH CELIAC DISEASE}

A case of clinical and electrophysiologic improvement following a glutenfree diet in a 34-year-old man with a 7-year history of slowly progressive ataxia is reported from the University of Napoli Federico II, Napoli, Italy. Neurologic exam revealed gait ataxia, positive Romberg's sign, nystagmus, dysarthria, dysmetria, and mild spasticity. Brain MRI showed vermis atrophy, and nerve conduction studies showed a sensory neuropathy. Vitamin E and B12 serum levels were normal. Antigliadin (AGAs) and antiendomysium antibodies (EMAs) were abnormal, and small bowel biopsy revealed total villous atrophy, crypt hyperplasia, and increased lymphocytes in the lamina propria. These signs of subclinical celiac disease had returned to normal when repeated 1 year later, following gluten-free diet. Neurological findings also improved after 2 years of dietary treatment. (Pellecchia MT, Scala R, Perretti A et al. Cerebellar ataxia associated with subclinical celiac disease responding to gluten-free diet. Neurology Oct 1999;53:1606-1608). (Reprints: Dr Paolo Barone, Clinica Neurologica, Ed 17, Department of Neurological Sciences, Via S Pansini 5, 80131 Napoli, Italy).

COMMENT. Cerebellar ataxia with classic celiac disease does not respond to the gluten-free diet, whereas the patient with subclinical celiac disease shows a remarkable improvement in neurologic signs and resolution of the villous atrophy.

Multiple cerebellar infarctions in a 7-year-old Japanese boy without cerebellar signs is reported from Shiga University, Hikone, Japan. (Ohno M, Suzuki A, Suzuki K et al. Cerebellar infarction in a young boy. Acta Paediatr Oct 1999;88:1162-1164). The child presented with uveitis, of undetermined cause. 